\title{
Gesellschaftliche Grenzregime der Moderne: das anthropologische Quadrat
}

\section{Gesa Lindemann}

\begin{abstract}
Die Theorie gesellschaftlicher Grenzregime führt die Frage nach den Grenzen von Vergesellschaftung mit der Frage nach der Differenzierung von Gesellschaften zusammen. Vergesellschaftung von ihren Grenzen her zu denken, heißt die Frage nach dem moralischen Status von Beteiligten zu stellen. Die Art und Weise, wie Gesellschaften diese Frage beantworten, hängt mit der Art ihrer Differenzierung zusammen. Der Beitrag skizziert die Struktur der modernen Grenzziehung zwischen Personen und anderen Wesen, untersucht das Verhältnis zwischen der Analyse gesellschaftlicher Grenzregime zu anderen Grenzregimeanalysen und arbeitet die Bedeutung des modernen gesellschaftlichen Grenzregimes für das normative Selbstverständnis der Moderne heraus.
\end{abstract}

\section{Schlagwörter}

Grenzen des Sozialen, anthropologisches Quadrat, Mensch, Staat

\section{Einleitung}

Es gehört zu den wichtigen Einsichten der Gesellschaftstheorie, dass Gesellschaft nicht mit staatlich begrenzten Gesellschaften gleichgesetzt werden kann. Bereits 1971 hatte Niklas Luhmann (vgl. 1971/2005) den Begriff „Weltgesellschaft“ eingeführt und darauf aufmerksam gemacht, dass die moderne, von ihm als funktional differenziert bezeichnete Gesellschaft nicht bzw. nicht ausschließlich mit Bezug auf den Staat begriffen werden könne. Vielmehr sei diese Gesellschaft in sachlich ausdifferenzierte Kommunikationssphären untergliedert: Wirtschaft, Politik, Recht, Wissenschaft usw. (vgl. Luhmann 1997, Bd. 2, S. 743ff.). Einzig das Rechtssystem sowie das politische System bzw. der Staat seien regional begrenzt, während die Systeme Wirtschaft und Wissenschaft nur als weltgesellschaftlich ausgreifende Funktionssysteme zu begreifen seien. Nicht die regionale und politische Begrenzung, sondern die weltgesellschaftliche Orientierung seien das zentrale Merkmal der modernen Gesellschaft. Es ist daher leicht einzusehen, dass die Grenzen von Gesellschaft nicht mit „Staatsgrenzen“ gleichgesetzt werden können (vgl. auch Schroer in diesem Band).

In der Gesellschaftstheorie wurden bislang die Grenzen des Kreises moralisch relevanter Akteure nicht explizit in den Blick genommen. Dies liegt daran, dass Gesellschaft implizit als Vergesellschaftung von Menschen begriffen wird. Damit folgt die Gesellschaftstheorie dem normativen Selbstverständnis der transatlantischen Moderne ${ }^{1}$, wonach im Rahmen des Ethos der Menschenrechte dem Menschen eine normativ herausgehobene Sonderstellung zukommt. Die Identifikation des Kreises moralisch relevanter Akteure mit dem Kreis der lebenden Menschen bleibt deshalb eine weitgehend unreflektierte Annahme und charakterisiert als solche den soziologischen Mainstream. Mit Bezug auf die ethnologische Forschung war diese Gleich-

1 Unter ,transatlantischer Moderne“ verstehe ich die Gesellschaftsordnung, die sich in Westeuropa und Nordameri$\mathrm{ka}$ in der Sattelzeit (Koselleck), d.h. zwischen 1750 und 1850, herausgebildet hat. Es ist eine empirische Frage, ob auch andere Weltregionen in diesem Sinne als modern zu bezeichnen sind. 
setzung schon seit Längerem fragwürdig geworden (für einen neueren zusammenfassenden Überblick vgl. Descola 2005/2011). Die Bedeutung der ethnologischen Einsichten für die Soziologie wurde nur zögerlich wahrgenommen. Thomas Luckmann (1970) gehörte zu den wenigen, der sich mit diesen Forschungen auseinandersetzte und kam zu dem Ergebnis, dass die Grenzen des Sozialen im Wesentlichen mit den Grenzen des Moralischen zusammenfallen. Sozialen Akteuren bzw. Personen komme ein moralischer Status zu. Sie können normative Erwartungen verletzen bzw. ihre normativen Erwartungen können von Personen verletzt werden. Man kann sich das an einem einfachen Beispiel verdeutlichen. Gegenwärtig gelten Bäume nicht als soziale Akteure. Wenn ein Baum auf einen Menschen fällt und dieser dadurch zu Tode kommt, so ist das kein moralischer Sachverhalt. Ein Baum ist nicht dazu in der Lage, die Norm zu verletzen, wonach Menschen nicht getötet werden sollen. Es wäre allerdings ein moralischer und auch ein rechtlicher Sachverhalt, wenn ein Mensch den Baum angesägt hätte, damit dieser auf einen anderen Menschen fällt. Ein Mensch kann die normative Erwartung, dass Menschen nicht getötet werden sollten, verletzen. Luckmanns zentrale Einsicht besteht darin, dass es Gesellschaften gibt, in denen nicht nur Menschen, sondern auch Tiere oder Pflanzen oder Geister bzw. Götter oder Gott als soziale Akteure zählen. Im Prinzip sei der Kreis derjenigen, die als moralisch relevante soziale Akteure gelten können, offen (vgl. auch Luckmann 1970; Kelsen 1946/1982; Descola 2005/2011), aber im Rahmen eines jeden Vergesellschaftungsprozesses wird dieser Kreis in einer verbindlichen Weise begrenzt.

Die Theorie gesellschaftlicher Grenzregime führt die Frage nach den Grenzen von Vergesellschaftung mit der Frage nach der Differenzierung von Gesellschaften zusammen. Es geht also um die Frage, wie die Begrenzung von Vergesellschaftung mit der Differenzierung von Gesellschaft zusammenhängt. Für die moderne Gesellschaft heißt das, die Begrenzung der Vergesellschaftung auf Menschen geht einher mit einer spezifischen Form gesellschaftlicher Differenzierung: der horizontalen Differenzierung. ${ }^{2}$

Dieses Verständnis gesellschaftlicher Grenzregime unterscheidet sich in charakteristischer Weise etwa von dem an Foucault angelehnten Grenzregimeverständnis, welches in den Analysen der europäischen Grenzsicherung in Anschlag gebracht wird (vgl. Hess et al. 2015; Hess/ Schmidt-Sembdner in diesem Band). Diese Analysen setzen voraus, dass es einen Kreis moralisch relevanter Wesen gibt, die ein Recht auf ein Leben in Freiheit und Würde haben, nämlich lebende Menschen. Wenn der Kreis moralisch relevanter Wesen mit dem Kreis der Menschen identisch ist, ist es kritikwürdig, wenn einige Menschen von den Möglichkeiten ausgeschlossen werden, innerhalb der EU ein Leben in Freiheit und Würde zu führen, sondern dem sicheren Tod ausgesetzt sind.

Damit klärt sich der Status der Theorie gesellschaftlicher Grenzregime im Verhältnis etwa zu foucaultschen Grenzregimeanalysen. Letztere setzen einen allgemeinen normativen Ordnungsrahmen voraus, ohne ihn als solchen zu thematisieren. Die Theorie gesellschaftlicher Grenzregime bezieht den allgemeinen normativen Ordnungsrahmen von Vergesellschaftung in die Analyse ein und rekonstruiert auf diese Weise auch die Bedingungen normativer Kritik an kon-

2 Horizontale Differenzierung beschreibt eine gesellschaftliche Ordnung, in der unterschiedliche Handlungsbereiche nicht in einem vertikalen, d.h. hierarchischen Verhältnis zueinanderstehen, sondern in einem horizontalen Verhältnis nebeneinanderstehen. Politik und Recht auf der einen Seite sowie etwa die Wirtschaft auf der anderen Seite stehen nicht in einem hierarchischen Verhältnis zueinander, sondern in einem Verhältnis wechselseitiger Abhängigkeit, ohne eindeutige Dominanz des einen oder anderen Bereichs. Parsons (1971/1985) und Luhmann (1997) sprechen von funktionaler Differenzierung. Für eine genauere Darstellung des Unterschieds zwischen horizontaler und funktionaler Differenzierung vgl. Lindemann (2018, S. 137f.; 2020, 63ff) 
kret ablaufenden Grenzziehungsprozessen innerhalb des Kreises moralisch relevanter Akteure. Auf diese Weise wird verständlich, warum es in modernen Gesellschaften skandalisiert werden kann, wenn vor Hunger und politischer Verfolgung fliehende Menschen im Mittelmeer nicht gerettet oder ethnische, religiöse oder sexuelle Minderheiten diskriminiert und verfolgt werden.

Der Kreis moralisch relevanter Akteure wird innerhalb der Theorie gesellschaftlicher Grenzregime als personaler Seinskreis bezeichnet. Es ist ein Kennzeichen der modernen Vergesellschaftung, dass der personale Seinskreis auf den Kreis der lebenden Menschen begrenzt wird. Die Grenzen des Menschlichen werden anhand einer vierfachen Abgrenzung gezogen, die ich als das „anthropologische Quadrat“ (vgl. Lindemann 2018, S. 101ff.) bezeichne. Hierzu gehören die Grenzen am Lebensanfang und am Lebensende sowie die Mensch-Tier- und die MenschMaschine-Grenze. Das Besondere dieser Grenzziehungen besteht darin, dass die Grenzen des Menschlichen mit den Grenzen des Sozialen zusammenfallen. Man kann sich dies verdeutlichen, indem man die Grenzen in den Blick nimmt, die in der europäischen Vormoderne noch relevant waren, etwa die abgrenzende Unterscheidung zwischen Menschen und Dämonen oder Teufeln. Diese waren relevant, wenn es um die Frage ging, ob ein Mensch von einem Teufel besessen war oder ob er einen Pakt mit ihm geschlossen hatte. Offensichtlich waren diese Grenzen des Menschlichen nicht identisch mit den Grenzen des Sozialen, denn es war möglich, mit dem Teufel einen Vertrag zu schließen, also eine soziale Beziehung einzugehen (vgl. Neumann 2007). Die moderne, d.h. die horizontal differenzierte Gesellschaft, scheint die erste zu sein, die die Grenzen des Sozialen eindeutig mit den Grenzen des Menschlichen identifiziert, sie ist gewissermaßen die erste Gesellschaft, die aus menschlichen Körperindividuen besteht. ${ }^{3}$

Die Ausbildung des anthropologischen Quadrats ist ein integraler Bestandteil der Entwicklung hin zur modernen Form gesellschaftlicher Differenzierung, der horizontalen Differenzierung. ${ }^{4}$ Die Theorie der horizontalen Differenzierung unterscheidet sich von derjenigen der funktionalen Differenzierung im Wesentlichen in zwei Punkten. Erstens: Analytisch kommt dem Institutionenbegriff eine zentrale Bedeutung zu und nicht wie in der Theorie funktionaler Differenzierung dem Systembegriff. Zweitens: Gegenstandsbezogen geht die Theorie funktionaler Differenzierung von einer Mehrzahl von Systemen aus, die nebeneinanderstehen. Im Unterschied dazu stellt die Theorie horizontaler Differenzierung die Differenzierung zweier Typen von Handlungszusammenhängen in den Mittelpunkt: zum einen mitgliedschaftlich verfasste und zum anderen weltgesellschaftlich orientierte Handlungs- und Kommunikationszusammenhänge (vgl. Lindemann 2018, S. 157ff.).

Erstens: Der in der Theorie horizontaler Differenzierung (vgl. ebd.) verwendete Institutionenbegriff schließt sowohl an Berger und Luckmann (1966/1980) als auch an den frühen Luhmann (1972) an. Danach sind Institutionen als typisierte Handlungsabläufe zu verstehen, die von typisierten Akteuren ausgeführt werden. Von besonderer Bedeutung ist dabei, dass Institutionalisierung reflexiv wird (vgl. Lindemann 2014, Kap. 3.4; 2018, S. 51f.). Damit ist Folgendes gemeint: Typisierte Handlungsabläufe, die von typisierten Akteuren ausgeführt werden, werden in einer typisierten Form miteinander gekoppelt. Die messbare Zeit ist eine solche vermittelnde Institution, die es erlaubt einzelne Handlungsabläufe miteinander zu koppeln. Um 7 Uhr Familienfrühstück, um 8 Uhr Arbeitsbeginn, um 16 Uhr Feierabend, um

3 Eine historische Rekonstruktion der Entwicklung hin zur menschlichen Gesellschaft findet sich bei Lindemann (vgl. 2018, Kap. 1).

4 Im Unterschied zur modernen Grenzziehung zeichnet sich etwa das vormoderne Europa dadurch aus, dass es noch keine strikten Grenzen des Sozialen, sondern eher kontextabhängige situative Grenzziehungen gibt (vgl. Descola 2005/2011; Lüdtke 2015). 
16.30 Uhr die Tochter aus dem Kindergarten abholen. Die exakt gemessene Zeit ermöglicht es, einzelne institutionalisierte Handlungsabläufe (Aufstehen, Familienfrühstück, Zur-ArbeitFahren, Arbeitsabläufe usw.) in eine institutionelle, d.h,. eine sicher erwartbare Reihenfolge zu bringen. Dies wird durch die gemessene Zeit erreicht, deshalb bezeichne ich sie als eine vermittelnde Institution. Reflexive Institutionalisierung kann auch mehrstufig funktionieren. Es gibt die Institutionen erster Stufe, die einzelne Handlungsabläufe strukturieren, es gibt Institutionen zweiter Stufe, die zwischen Institutionen der ersten Stufe vermitteln. Es kann aber auch noch höherstufige Institutionen geben, die etwa sinnhafte Zusammenhänge zwischen Institutionen erster und zweiter Stufe herstellen. Legitimationstheorien im Sinne von Berger und Luckmann wären solche Institutionen dritter Stufe: Sie stellen Zusammenhänge zwischen sehr verschiedenen Institutionen erster und zweiter Stufe her. So können etwa die Herstellung unterschiedlicher Güter und deren Verkauf bzw. Kauf als ein zusammenhängender Handlungszusammenhang beschrieben werden, nämlich als gewinnorientierte Wirtschaft. Wenn solche legitimierenden Beschreibungen praktisch wirksam werden, werden ausdifferenzierte Handlungszusammenhänge, wie eben die gewinnorientierte Wirtschaft, institutionalisiert.

Zweitens: Die Theorien menschlicher Würde (Kant) bzw. der Menschenrechte (Allgemeine Erklärung der Menschenrechte) sind noch weiter ausgreifende Legitimationstheorien, die lebende Menschen als die einzigen Wesen mit einem vollen moralischen Status beschreiben. Sie funktionieren praktisch im Sinne einer konkreten handlungsrelevanten institutionellen Festlegung, die bestimmt, welche Wesen in dieser Weise anzuerkennen sind. Der institutionell gesteuerte Prozess, in dem lebende Menschen in dieser Weise zu Personen werden, ist die mitgliedschaftliche Erfassung aller lebenden Menschen durch die einzelnen Nationalstaaten. Mitgliedschaftliche Verfasstheit ist konstitutiv für die Zusammenhänge von Politik und Recht, während eine weltgesellschaftliche Orientierung vor allem in den Handlungszusammenhängen von Wirtschaft und Wissenschaft realisiert ist.

Die Besonderheit mitgliedschaftlich verfasster Handlungs- und Kommunikationszusammenhänge in den Mittelpunkt zu rücken, führt auf die Bedeutung der ungeheuren, maßgeblich vom Staat getragenen Organisationsleistung, durch die sich moderne Vergesellschaftung auszeichnet. Staatliche Instanzen sorgen dafür, dass mehr oder weniger vollständig jede Geburt und jeder Todesfall organisatorisch erfasst und dokumentiert wird. Die staatliche Kontrolle der Aufnahme neuer Mitglieder bzw. ihres Ausscheidens wird vermittels der Medizin, d.h. der Medikalisierung von Schwangerschaft, Geburt, Sterben und Tod, konkret durchgesetzt. ${ }^{5}$ Diejenigen, die in dieser Weise als Staatsbürger organisatorisch erfasst werden, gelten als Menschen mit universalen Menschenrechten, ihnen kommen Freiheit und Würde zu. Aufgrund dieses staatlich bzw. rechtlich garantierten Status sind Bürger als Menschen freigestellt, sich global zu vergesellschaften. Zum Beispiel: Für die Ausbildung des global ausgreifenden Handlungsund Kommunikationszusammenhangs Wirtschaft ist es notwendig, dass sich Menschen frei bewegen und frei über ihr Eigentum verfügen können. Dies ermöglicht den Menschen, sich als Marktteilnehmer global zu vergesellschaften, indem sie etwas kaufen oder verkaufen (vgl. Lindemann 2018, Kap. 2.6).

Die Institution des Menschen, gleich an Freiheit und Würde in den Grenzen des anthropologischen Quadrats, beschreibt einen in sich spannungsvollen Sachverhalt. Die geborenen Körper-

5 Dieser Aspekt wird in der soziologischen Literatur zumeist kritisch als Herrschaftssicherung analysiert (vgl. u.a. Foucault 1963/1988; Duden et al. 2002; Bergmann 2005). Dass es sich dabei um eine für die Herausbildung des modernen Körperindividualismus relevante Praxis handelt, wird zumeist übersehen. 
individuen werden zwar staatlich erfasst, aber nicht darauf beschränkt, Glieder ihres Staates zu sein. Vielmehr garantiert der Staat einen Rechtsstatus, der den Bürger zum Menschen macht, dem es freisteht, sich in beliebige Handlungs- und Kommunikationszusammenhänge (Wirtschaft, Recht, Wissenschaft usw.) zu involvieren. Zentral hierfür sind die verfassungsmäßig garantierten Grundrechte. Durch diese begrenzt sich der Staat bzw. die Politik in seinem/ihrem Zugriff auf die menschlichen Körperindividuen (vgl. Luhmann 1965/1999; Lindemann 2018, Kap. 3).

Die Grenzen des anthropologischen Quadrats beschreiben zwei unterschiedliche Arten von Grenzen. Die Grenzen am Lebensanfang und am Lebensende sind überschreitbar. Sie werden durch konkrete Organisationsleistungen bzw. grenzsichernde Praktiken stabilisiert. Die Grenzen zwischen Mensch und Maschine sowie Mensch und Tier bezeichnen zwei zur Zeit noch unüberschreitbare Grenzen. Dennoch sind diese Grenzen relevant für das diskursiv verhandelte menschlich-moralische und damit auch das politische Selbstverständnis der horizontal differenzierten Vergesellschaftung.

Maschine

Lebensanfang

Mensch/Person

Lebensende

gleich an Freiheit und Würde

Tier

Abb. 1: Das anthropologische Quadrat

\section{Das anthropologische Quadrat}

Der zweigeschlechtlich verkörperte moderne Mensch gleich an Freiheit und Würde ist das institutionelle Element, aus dem moderne Gesellschaften aufgebaut sind. Dieser Sachverhalt ist nicht einfach zu verstehen, denn nicht nur der Sachverhalt, dass dem Menschen Freiheit und Würde zukommen, ist ein institutioneller Sachverhalt, sondern auch, dass der Mensch ein natürliches Wesen und damit ein diesseitig lebendiger Körper ist, muss als eine Institution verstanden werden. Als ein Körper, der im Sinne der naturwissenschaftlichen Biologie zu erforschen ist, beginnt der Mensch zu leben und stirbt (Grenzen am Lebensanfang und am Lebensende), als von der Biologie zu erforschender diesseitiger natürlicher und gemäß Naturgesetzen existierender Körper könnte der Mensch ein Tier sein oder auch ein technisch herzustellendes Ding wie ein Roboter. Diese Unterscheidungen sind nicht einfach vorhanden, vielmehr handelt es sich um umstrittene Unterscheidungen, deren Gültigkeit immer wieder in Frage gestellt wird. Die Grenzen des anthropologischen Quadrats sind nicht einmalig fixierte Grenzen, vielmehr wird es immer wieder fraglich, ab wann ein sich entwickelnder menschlicher Embryo bzw. Fötus als ein personales Wesen mit einem Recht auf Leben zu gelten hat. Es wird immer wieder fraglich, ab wann ein Mensch tot ist. Und es wird immer wieder in Frage gestellt, ob es tatsächlich eine Grenze zwischen Mensch und Tier bzw. Mensch und Maschine 
gibt. Die Grenzen des anthropologischen Quadrats existieren, insofern sie dramatisiert und als problematische Grenzziehungen inszeniert werden, d.h., diese Grenzen gibt es nicht als einmalig fixierte und im Weiteren selbstverständlich geltende Grenzen. Vielmehr werden die Grenzen des anthropologischen Quadrats immer wieder in öffentlichen Debatten in Frage gestellt. Auf diese Weise wird die diskursive Problematisierung der Grenze immer wieder dramatisch in Szene gesetzt. Bis jetzt haben alle dramatischen Problematisierungen immer wieder zu einer Stabilisierung der Grenzziehung geführt. Solche Debatten finden sich mit Bezug auf alle vier Grenzen. Die Debatten um Todesfeststellung und Abtreibung begleiten die Moderne ebenso wie die Debatten darum, ob es möglich ist, menschenähnliche Maschinen herzustellen, oder ob es tatsächlich einen qualitativen Unterschied zu Tieren gibt.

\subsection{Lebensanfang}

Die institutionell relevante Grenze am Lebensanfang ist die als natürlich verstandene Geburt. Diese neue Form der Grenzziehung basiert auf einer staatlichen Organisationsleistung. Der Staat erfasst und dokumentiert die Geburt bzw. die Geborenen - vermittelt über die Medizin. Diese staatlichen Organisationsleistungen, durch die die Grenzen am Lebensanfang gezogen werden, haben einen Vorläufer: die Taufe. Durch die Taufe wurde zum ersten Mal in der europäischen Geschichte ein einheitlicher Rechtsstatus geschaffen (vgl. Meyer 2013). Es wäre eine ausführliche vergleichende Forschung erforderlich, um zu prüfen, ob es vergleichbare Rituale auch in anderen Kulturen gibt. Es scheint, als würden die anderen großen Weltreligionen Rituale, die einen einheitlichen Rechtsstatus verleihen, nicht kennen. Eine entsprechende vergleichende religionssoziologische Forschung gibt es kaum. Mit der Durchsetzung der Taufe bzw. durch deren Verbindung mit der Namensgebung wurde die Macht der Familien über die Geborenen drastisch beschnitten. Es lag nun nicht mehr in der Gewalt der Familie, die Neugeborenen aufzunehmen bzw. sie auszustoßen oder zu töten. Vielmehr wurde es seit der Zeit zwischen dem 11. und 13. Jahrhundert Sache der Kirche, die Neugeborenen offiziell als Glied der christlichen Gemeinschaft aufzunehmen. Dies konnte nur gelingen, weil die Kirche zu einer entsprechenden Organisationsleistung in der Lage war. Für Pfarrer wurde die Anwesenheit in ihrer Gemeinde zunehmend Pflicht. Sie wurden von höheren Stellen immer wieder angehalten, alle Neugeborenen zu taufen und dies entsprechend in den Taufbüchern zu dokumentieren (vgl. Lindemann 2018, S. 85ff.). In diesem Rahmen entwickelte sich ein auf die unsterbliche Seele und ihr Verhältnis zu Gott bezogenes Individualitätsverständnis: der Seelenindividualismus. Aus Wesen, die primär durch ihre Familienbeziehungen bestimmt waren, wurden Seelenindividuen (vgl. ebd., Kap. 1).

Im Verlauf der Sattelzeit (1750-1850) übernahmen die modernen Staaten zunehmend die zuvor von der Kirche ausgeübte Kontroll-, Erfassungs- und Dokumentationspraxis. Damit veränderten sich sowohl der organisationale als auch der identitätsbildende Bezugspunkt für die Ausbildung des Selbstverständnisses als dauerndes Individuum, welches für seine Taten verantwortlich gemacht werden kann. Nicht mehr die Seele, sondern der als natürlich verstandene Körper, d.h. der Körper als ein dreidimensional ausgedehntes und Naturgesetzen unterworfene Ding, wird zum Bezugspunkt der Individualisierung. Das entscheidende Kennzeichen dieser Veränderung bestand darin, dass im Laufe des 19. Jahrhunderts die Taufe als Bedingung der vollen Rechtsfähigkeit durch eine naturalisierte Bedingung abgelöst wurde: die Geburt. 
Diese wurde nicht in einem Kirchenbuch dokumentiert, sondern in einem von staatlicher Seite geführten Personenstandsbuch (vgl. ebd., S. 114ff.).

Sowohl bei der Geburt als auch bei der Taufe handelt es sich um einen institutionellen Sachverhalt. Dessen Sinn besteht darin, die Betreffenden aus dem familialen Zusammenhang heraus zu individualisieren, d.h., den Betreffenden wird die Bildung eines Selbstverständnisses als Individuum jenseits familialer Zusammenhänge zugemutet. Die Taufe bezieht den Täufling in die Gemeinschaft der Christen ein und setzt ihn als Seelenindividuum in Bezug zu Gott. Dies sicherte dem Christen einen Rechtsstatus, der durch die irdische Gemeinschaft nicht mehr annulliert werden konnte. Sogar nach der Exkommunikation blieben die Betreffenden innerhalb der Christengemeinschaft. Die moderne Geburt sichert dem Menschen als Staatsbürger den Rechtsstatus als Individuum und mutet ihm zu, sich als menschliches Körperindividuum zu verstehen. Nicht mehr die Anerkennung als Seelenindividuum, sondern die Anerkennung als Körperindividuum sichert dem Neugeborenen einen Rechtsstatus, der es aus der Familie löst; seine Rechtsfähigkeit hängt nicht von der Anerkennung durch Vater oder Mutter ab. Diese haben nicht das Recht, das Neugeborene zu töten, wie es im Rahmen vorchristlicher gentiler, d.h. familienbezogener, Rechte der Fall war. Im Übergang zur Moderne wird der allgemeine Rechtsstatus gegen die Familie durch die Staatsunmittelbarkeit der dokumentierten Geburt gesichert.

Sowohl im Rahmen des christlichen als auch im Rahmen des modernen individuellen Rechtsstatus als Person stellt sich die Frage, ob er auf die Phase vor der Geburt ausgedehnt werden soll. Soll die Rechtsgarantie, die die individuelle Seele bzw. den individuellen lebendigen menschlichen Körper aus dem familiären Zusammenhang löst, bereits vor der Geburt in Geltung gesetzt sein? Solange das Taufsakrament entscheidend für das Verständnis als personales Individuum jenseits der Familie war, standen die Seelen im Mittelpunkt. Aus diesem Grund musste bei der vorgeburtlichen Individualisierung durch die Taufe das irdische Überleben der Schwangeren nicht unbedingt in Rechnung gestellt werden. Diese war bereits Teil der christlichen Gemeinschaft, die auch über den Tod hinaus dauerte. Das Versterben der schwangeren Frau war insofern kein problematischer Ausschluss aus dem Kreis der Personen (vgl. ebd., S. 91ff.). Dies änderte sich, als die individualisierende Anerkennung mit Bezug auf den diesseitig lebendigen Menschen erfolgte, denn jetzt wäre der Tod der Schwangeren ein endgültiger Ausschluss aus dem Kreis anerkannter Personen (s.u. den Abschnitt ,Lebensende`). Es entstand also eine normativ hoch problematische Konfliktsituation, die zugunsten der Schwangeren aufgelöst wurde und wird. Im Konfliktfall soll das bereits anerkannte personale Leben der schwangeren Frau mehr gelten als das Leben des Fötus, der noch nicht als Person mit einem vollen moralischen Status anerkannt ist. Wie die Gewichtung im Einzelnen erfolgen soll, bleibt umstritten. Der Streit um die Abtreibung gehört zu großen Debatten, die die Moderne seit dem 19. Jahrhundert begleiten (vgl. Graumann 2012). Grundsätzlich resultiert aus dieser Gewichtung, dass das Leben des Fötus zwar verhandelbar ist, aber dennoch in abgestufter Weise unter Schutz steht.

Unabhängig von solchen Debatten hat sich mit der Entwicklung der Frühgeborenen-Intensivmedizin ein praktisches Konfliktfeld entwickelt. Aber auch hier bleibt die Grenze der Geburt erhalten. Solange ein Fötus sich im Mutterleib befindet, ist sein Leben grundsätzlich verhandelbar. In Deutschland kann bis kurz vor der regulären Geburt noch eine Spätabtreibung durchgeführt werden. Wenn ein Fötus aber aus dem Mutterleib heraus ist, auch wenn er früh geboren ist, ist er eine rechtsfähige Person, der grundsätzlich ein Recht auf Leben und damit 
auf medizinische Behandlung zukommt (vgl. Büsing 2021; Schaeffer 2021). Damit ist die faktisch existierende Grenze des anthropologischen Quadrats am Lebensanfang ausgemacht. Ein heranwachsender menschlicher Körper ist dann eine Person, wenn er sich außerhalb des mütterlichen Körpers befindet, in dem er herangewachsen ist. Sowie er geboren ist, hat ein menschlicher Körper das Recht auf eine, notfalls invasive, intensivmedizinische Behandlung so ist die gegenwärtige gesellschaftliche Praxis.

\subsection{Lebensende}

Mit dem Übergang in die moderne Form der Vergesellschaftung setzte sich auch ein neues Verständnis des Sterbens bzw. des Todes durch. Danach wurde das Eintreten des Todes nicht mehr mit dem Zeitpunkt identifiziert, an dem die Seele den Körper verlässt. Vielmehr wird der Tod zu einem material identifizierbaren Zerfallsprozess des Lebens (vgl. Pernick 1988, S. 28ff.; Rüve 2008, S. 63f.). Wenn die Seele nicht mehr das tragende Prinzip des Lebens ist, sondern der Körper, endet mit dem diesseitigen Leben das Leben überhaupt. Der für die christliche europäische Vormoderne prägende Gedanke der Wiederauferstehung verlor grundsätzlich an Evidenz, denn das Leben wurde in allgemeinverbindlicher Weise ausschließlich an den diesseitigen Körper gebunden. Mit dem Tod war das Leben beendet. Wenn der Tod grundsätzlich als unwiderruflicher Ausschluss aus dem Kreis sozialer Personen verstanden wird, wird die Frage nach sicheren Todeszeichen zu einem Problem. Solange die Möglichkeit der Widerauferstehung existierte, war die Grenze zwischen Leben und Tod grundsätzlich in beide Richtungen passierbar. Es war auch möglich, dass Totengeister den Lebenden zusetzten und beruhigt werden mussten (vgl. Rüve 2008, S. 56). Wenn der Mensch nur noch ein körperliches Leben hat, gibt es nur noch eine mögliche Richtung des Grenzübertritts. Vorzeitig begraben zu werden, ist nicht wirklich ein Problem, wenn ohnehin die Hoffnung auf die Wiederauferstehung besteht. Wenn der Tod allerdings endgültig das Ende bedeutet, wird es zu einem erheblichen Problem, vorzeitig beerdigt zu werden. Die Debatte um den Scheintod, die im 18. Jahrhundert begann und bis in das 19. Jahrhundert andauerte (vgl. ebd., S. 9), dokumentierte die Probleme, die sich aus der Verkörperung und damit der neuen Endgültigkeit des Todes ergaben (vgl. Kessel 2001, S. 136).

Die Lösung des Scheintodproblems erfolgte durch eine weitere beachtliche Organisationsleistung: die staatlich-bürokratisch forcierte Einbeziehung der Medizin in die Todesfeststellung. Während die Mediziner vor der Sattelzeit den Sterbenden fernbleiben sollten, wurden sie jetzt staatlicherseits verpflichtet, den Tod festzustellen (vgl. Kessel 2001, S. 149f.). Um 1900 hatte sich das Scheintodproblem weitgehend aufgelöst - zum einen aufgrund der als sicher geltenden medizinischen Todesfeststellung und zum anderen aufgrund der staatlich durchgesetzten Praktiken des Aufbahrens von Toten vor der Beerdigung (vgl. Pernick 1988, S. 31, 35). Erst in den 1950er Jahren entstanden neue Unsicherheiten. Im Mittelpunkt standen einerseits die Entwicklung der Intensivmedizin, vor allem die Techniken der maschinell gestützten künstlichen Beatmung und der künstlichen Aufrechterhaltung des Blutkreislaufs, und andererseits die Erfolge der Reanimationsforschung, der es gelang, im Experiment auch höhere Säugetiere nach allen Regeln der Kunst zu töten und vollständig wieder zu beleben (vgl. Lindemann 2003, S. 55 ff.). Die neuen Techniken der Lebenserhaltung bzw. die Reanimationsforschung bildeten die unmittelbare Voraussetzung der Hirntodkonzeption, zugleich stellte die Entwicklung der 
Hirntodkonzeption einen Scheidepunkt dar. Durch die Intensivmedizin und die Reanimationsforschung wurde die Unsicherheit hinsichtlich der Todesfeststellung auf die Spitze getrieben. Die neuen Techniken ließen es sogar als möglich erscheinen, den Tod endgültig zu überwinden (vgl. ebd., S. 67f.). Erst die Etablierung der hirnbezogenen Todesfeststellung beendete die hoffnungsvolle Unsicherheit, den Tod technisch beherrschen zu können. Das Hirntodkonzept war zunächst ein Mittel, das es erlaubte, die Sinnlosigkeit einer weiteren Behandlung zu begründen (vgl. ebd., S. 69ff.), und es führte schließlich erneut zu der Vorstellung, den Tod definitiv feststellen zu können (vgl. ebd., S. 74ff.). Erst als der Hirntod seit dem Ende der 1960er Jahre als Bedingung für die Entnahme transplantierbarer Organe begriffen wurde (vgl. ebd., Kap. 2.2.1.), entstand erheblich zeitverzögert in den 1990er-Jahren erneut eine intensive öffentliche Dramatisierung der Grenze am Lebensende (vgl. Fuchs 1996), die bis in die Gegenwart, d.h. 2020, anhält.

\subsection{Mensch-Tier}

Zwischen den Grenzziehungen am Lebensanfang und am Lebensende sowie den Grenzziehungen Mensch-Maschine und Mensch-Tier gibt es wichtige Unterschiede. Die beiden erstgenannten Grenzziehungen bezeichnen überschreitbare Grenzen. Das heißt, aus einem Wesen, das noch keinen vollen personalen Status hat (Embryo, Fötus), wird ein Wesen mit einem vollen personalen Status, aus dem am Lebensende ein Wesen wird, das keinen vollen personalen Status mehr hat, ein Leichnam. Die Grenzen am Lebensanfang bzw. -ende sind insofern von praktischer Relevanz und erfordern umfangreiche Organisationsleistungen, die die Grenzziehung sichern. Im Unterschied dazu steht bei den (noch) unüberschreitbaren Grenzen zwischen Mensch und Tier sowie Mensch und Maschine der Diskurs über das menschliche Selbstverständnis im Mittelpunkt.

Was die Unterscheidung zwischen Mensch und Tier betrifft, war seit der zweiten Hälfte des 19. Jahrhunderts die Auseinandersetzung um Charles Darwins Werk von zentraler Bedeutung. Der historische Ablauf im Übergang zur Sattelzeit stellt sich wie folgt dar: Der diesseitige verkörperte Mensch entsteht in der Sattelzeit und bekommt von Darwin (1871) eine Jahrtausende währende Entwicklungsgeschichte in die Wiege gelegt.

Trotz ihrer Unterschiedlichkeit gibt es eine wichtige Gemeinsamkeit zwischen der Grenze Mensch/Tier und den Grenzen am Lebensanfang und am Lebensende. Es handelt sich nicht um eine einmalig fixierte Grenze, vielmehr wird diese Grenze immer wieder diskursiv dramatisiert. In der diskursiven Dramatisierung lässt sich eine in sich widersprüchliche normative Struktur identifizieren, denn die Unterscheidung zwischen Mensch und Tier kann in zwei Richtungen problematisiert werden. Zum einen kann es heißen, dass Tiere wie Menschen sind, weshalb auch Tieren entsprechende Rechte zuerkannt werden müssen, und zum anderen kann es heißen, dass Menschen wie Tiere sind, die sich zivilisieren müssen bzw. Menschen können zu tierähnlichen Wesen gemacht werden. Aus diesen Orientierungen ergeben sich unterschiedliche normativ aufgeladene Diskursstränge.

1) Tiere sind wie Menschen bzw. es gibt zumindest einige Tiere, die wie Menschen sind.

Dies führt zu einer inklusiven Schlussfolgerung: Wenn es Tiere gibt, die wie Menschen sind, müssen sie einen den Menschen ähnlichen normativen Status haben. 
2) Menschen sind (noch) wie Tiere und müssen sich erst zu Menschen entwickeln bzw. Menschen können zu tierähnlichen Wesen gemacht werden.

Hier ergeben sich zwei unterschiedliche moralische Schlussfolgerungen:

a) Die normative Mensch-Tier-Differenz: Es muss verhindert werden, dass Menschen zu Tieren gemacht bzw. degradiert werden.

b) Die pädagogisierte Mensch-Tier-Differenz: Wenn Menschen wie Tiere sind bzw. solange sie sich in einem tierähnlichen Zustand befinden, erübrigt sich die Orientierung am Ethos der Menschenrechte. Dies gilt zumindest für diejenigen Menschengruppen, die (noch) wie Tiere sind.

Die inklusive Schlussfolgerung bezieht sich auf das Problem, ob höher entwickelte Tiere nicht auch die Fähigkeiten haben, die den Menschen zum Menschen machen, weshalb ihnen den Menschenrechten analoge Rechte zugesprochen werden müssten (vgl. Cavalieri/Singer 1993/1996, vgl. auch die Netzseite des „Great Ape Projects“, www.greatapeproject.de).

Die zweite Frage bezieht sich darauf, ob Menschen wie Tiere sind oder ob sie zu Tieren gemacht werden können und welche moralischen Implikationen sich daraus ergeben. Die sich entwickelnde Biotechnologie hat zur Diskussion im Sinne der normativen Mensch-Tier-Differenz geführt. Hier geht es um die Frage, ob Menschen zu Tieren werden bzw. was es für die Frage der Tier-Mensch-Unterscheidung bedeutet, wenn Zellen oder Teile von tierischen Zellen zu Heilungszwecken in menschliches Gewebe bzw. Zellen eingebracht werden. Hier geht die Vermischung von menschlichem und tierischem Erbgut für einige soweit, dass auf diese Weise die Mensch-Tier-Grenze in Frage gestellt wird (vgl. Rothhaar et al. 2017). Ein Vorgang, den es den Kritikern zufolge auf jeden Fall zu verhindern gelte.

Insgesamt stellt es sich bislang so dar, dass solche Technologien zwar zu intensiven Debatten führen, ohne dass dadurch die Tier-Mensch-Grenze ernsthaft in Frage gestellt wird. Die Funktion von Diskursen über die Differenzen von Menschen und Affen und deren Rechte bzw. um die Biotechnologie besteht eher darin, durch die dramatische Infragestellung die Bedeutung dieser Grenze zu aktualisieren.

Die Thematisierung der Mensch-Tier-Grenze im Sinne der Orientierung an der pädagogisierten Mensch-Tier-Differenz war dagegen durchaus von praktisch politischer Relevanz. Sie prägte die Diskussion um das Verhältnis von Naturzustand und dem Grad von Zivilisation, den Menschengruppen erreicht haben. Danach entwickeln sich Menschen von einem den Tieren ähnlichen Naturzustand weg und hin zu einem immer höher zivilisierten wahrhaft menschlichen Zustand. Kultur in diesem Verständnis sei eine kollektive Schöpfung des Menschen, „die einer fortschreitenden Bewegung zur Vervollkommnung unterliegt“ (vgl. Descola 2005/2011, S. 121). In dieser Perspektive werden Gesellschaften daraufhin untersucht, wie sie sich vom Naturzustand entfernen und inwiefern ihre jeweiligen kulturellen Institutionen eine zunehmende Vervollkommnung zeigen.

Politisch dominierte vor allem im letzten Drittel des 19. Jahrhunderts die Doktrin, wonach die weißen Europäer eine besonders weit zivilisierte Menschengruppe wären, woraus praktisch ein Erziehungsauftrag resultierte. In der Praxis des Kolonialismus und in der Praxis der Erziehung der Unterschichten zur zivilisierten Disziplin (vgl. Foucault 1975/1979, Kap. III, Disziplin) zeigte sich, dass dazu auch Mittel erforderlich waren, die gegenüber bereits zivilisierten Menschen nicht mehr angewendet werden durften. Jedenfalls würde eine übertriebene Orientierung 
am Ethos der Gleichheit aller Menschen die Zivilisierung der sich noch im Naturzustand befindenden, eher noch tierähnlichen Menschen, nur behindern.

Das Fortschrittsmodell der Kultivierung und der daraus resultierende Zivilisationsauftrag gerieten in die Kritik, weil sie dazu gedient hätten und dienen würden, den Anspruch auf Überlegenheit und Führung von weißen Europäern und Amerikanern gegenüber den kolonisierten Ländern Afrikas und Asiens zu legitimieren und durchzusetzen (vgl. Said 1978).

In der Zeit nach dem Zweiten Weltkrieg verlor die Orientierung an der pädagogisierten Mensch-Tier-Differenz, wonach Menschen bzw. Menschengruppen zumindest vorübergehend wie Tiere sind und erst zu Menschen gemacht werden müssen, zunehmend an Bedeutung. Die beiden anderen Diskursstränge, d.h. Diskursstrang 1 und 2a, gewinnen dagegen an Bedeutung, wonach Tiere bzw. einige Tiere wie Menschen sind (Diskursstrang 1) bzw. wonach Menschen durch medizinische oder biotechnologische Eingriffe nicht den Tieren angeähnelt werden dürfen (Diskursstrang 2a, normative Mensch-Tier-Differenz). Es ist eine offene empirische Frage, ob die Orientierung an der pädagogisierten Mensch-Tier-Differenz grundsätzlich einen Bestandteil der möglichen diskursiven Dramatisierungen des anthropologischen Quadrats bildet. Wenn dem so wäre, kann vermutlich immer wieder an den entsprechenden Diskursstrang angeschlossen werden.

\subsection{Mensch-Maschine}

Auch im dramatisierenden Diskurs bezüglich der Mensch-Maschine-Grenze geht es immer wieder um das moralische Selbstverständnis des Menschen. Gemäß der diskursiven Dramatisierung gilt, wenn der Mensch ihm ähnliche künstliche Wesen schafft, muss er sie auch anerkennen. Wenn es sich um Wesen handelt, die wie Menschen sind, müssen sie auch wie Menschen behandelt werden. Das heißt, ihr Status muss sich an dem Ideal der Gleichheit aller Menschen und damit am Gebot der Allinklusion orientieren, denn wenn allen Menschen ein einheitlicher Rechtsstatus zukommt, muss dieser auch für Roboter gelten, wenn sie ausreichend genug den Menschen ähneln. Folglich müssen diese Wesen als Teil der menschlichen Gesellschaft anerkannt werden. Wenn die künstlichen Wesen von ihrer Natur her den Menschen nicht gleichen, gilt für sie auch das Gebot der Allinklusion nicht.

Die sich daraus ergebende Struktur der normativen Probleme ähnelt derjenigen, die sich bei der Mensch-Tier-Grenze gezeigt hat. Es gibt auch hier zwei unterschiedlich gelagerte, aber ebenfalls in sich widersprüchliche Orientierungen. Wenn das Wesen des Menschen problematisiert wird, indem die Mensch-Maschine-Grenze in Frage steht, kann dies in zwei Richtungen geschehen. Zum einen kann es heißen, dass der Mensch Maschinen herstellt, von denen zumindest einige wie Menschen sind, weshalb ihnen entsprechende Rechte zuerkannt werden müssen (inklusive Mensch-Maschine-Differenz) und zum anderen kann es heißen, dass Menschen allgemein im Sinne eines maschinellen Funktionierens zu begreifen sind, demnach sind Menschen wie Maschinen bzw. einige oder fast alle Menschen können zu maschinenähnlichen Wesen gemacht werden (degradierende Mensch-Maschine-Differenz). Aus beiden normativen Orientierungen können jeweils zwei unterschiedliche, normative Schlussfolgerungen abgeleitet werden. 
1. Maschinen sind wie Menschen bzw. es können Maschinen gemacht werden, die wie Menschen sind. Die inklusive Mensch-Maschine-Differenz offenbart zwei Probleme: das Hybrisproblem und das Anerkennungsproblem.

a) Hybrisproblem: Es stellt ein Problem dar, wenn Menschen sich in dieser Weise zum Schöpfer aufschwingen, denn es besteht die Gefahr, dass die Maschinen die Macht übernehmen.

b) Anerkennungsproblem: Wenn menschenähnliche Maschinen gebaut werden, muss ihnen ein normativer Status zuerkannt werden, der demjenigen von Menschen entspricht.

2. Menschen sind wie Maschinen bzw. Menschen können zu maschinenähnlichen Wesen gemacht werden. Die degradierende Mensch-Maschine-Differenz führt ebenfalls auf zwei unterschiedlich gelagerte Probleme.

a) Dies muss verhindert werden. Menschen dürfen niemals zu maschinenähnlichen Wesen degradiert werden. Wenn Menschen maschinenähnlich gemacht worden sind, müssen sie wieder zu Menschen gemacht, d.h. emanzipiert werden. Man kann dies als die normativ orientierte degradierende Mensch-Maschine-Differenz bezeichnen.

b) Wenn Menschen wie Maschinen sind, erübrigt sich die Orientierung am Ethos der Menschenrechte. In diesem Fall kann man von einer rein kognitiv orientierten degradierenden Mensch-Maschine-Differenz sprechen, denn es gilt die Tatsache anzuerkennen, dass Menschen wie Maschinen sind.

Die inklusive Mensch-Maschine-Differenz untergliedert sich in zwei Gruppen von normativen Problemen. Das Hybrisproblem, d.h., dass Menschen Maschinen schaffen, die wie Menschen sind, wird primär in der Science-Fiction-Literatur und dem Feuilleton verhandelt. ${ }^{6}$ Hier geht es darum, dass die Maschinen die Macht übernehmen und die Menschen unterwerfen. Eine stärker normativ aufgeladene Kritik ist religiös inspiriert. Hier geht es explizit darum, dass es menschliche Hybris wäre, solche Maschinen zu schaffen (vgl. Klinkhammer 1997).

Die zweite Gruppe bilden die normativen Anerkennungsprobleme, wonach Maschinen, welche wie Menschen sind, einen menschenähnlichen normativen Status haben müssten. Dies ist einerseits ebenfalls ein beliebtes Thema im Bereich Science-Fiction ${ }^{7}$, andererseits werden derzeit tatsächlich Maschinen entwickelt, die bislang von Menschen ausgeübte Tätigkeiten derart ausüben, dass sich bereits praktisch die Frage nach ihrem normativen Status stellt. Ein Beispiel hierfür sind Softbots, die im automatisierten Börsenhandel eigenständig Verträge abschließen, oder Roboter, die ihre Reaktionen auf die Umwelt durch einen Lernprozess verändern (vgl. Matsuzaki et al. 2016). Bei solchen künstlichen Wesen stellt sich die Frage, wie Verantwortung zugerechnet werden kann, wenn eine solche Maschine einen Schaden anrichtet (vgl. Matthias 2004). Für Fälle derartiger Verantwortungsübernahmen im Rahmen von Haftungsproblemen wird derzeit z.B. ein neuer rechtlicher Status diskutiert, der Status der ,Elektronischen Person (vgl. Beck 2016). Hierbei handelt es sich um eine künstliche Person, analog zur juristischen Person. Die elektronische Person würde registriert werden und wäre selbst versichert. In die Versicherung würden alle Herstellerinnen und evtl. die Nutzerinnen einzahlen. Die Statusbesonderheit der elektronischen Person besteht darin, dass es sich nicht um eine menschliche Person handelt, sondern um eine funktionsspezifische Personalitätskonstruktion. Die elektro-

6 Um nur einige Beispiele zu nennen: I, Robot (2014); The Matrix (1999); Demon Seed (1977); Der Golem, wie er in die Welt kam (1920).

7 Vgl. Alex Garland (2014) „Ex Machina“, Film mit Oscar Isaac und Domhnall Gleeson. 
nische Person ist Person in einer besonderen Hinsicht: Sie kann zivilrechtlich für einzelne Aktionen, die im Anwendungsbereich dieser Maschine liegen, zur Verantwortung gezogen werden. Dieser Weg weist eine interessante Richtung auf, wie Maschinen einen rechtlichen Status erhalten können, ohne dadurch die Mensch-Maschine-Grenze in ihrer faktischen Gültigkeit in Frage zu stellen (vgl. Matsuzaki/Lindemann 2016).

Die zweite Fragerichtung unterteilt sich ebenfalls in zwei klar voneinander zu unterscheidende Themencluster. Der normativen Problematisierung der degradierenden Mensch-Maschine-Differenz zufolge müsse es verhindert werden, dass Menschen zu maschinenähnlichen Wesen werden. Diese Themen werden in der romantischen Technikkritik (vgl. Klages 1913/2006) verarbeitet und bilden auch einen wichtigen Bestandteil der marxistischen Verdinglichungskritik (vgl. Marx 1890/1977). Demnach schaffen die Menschen Automatismen in ihren Beziehungen, in dem sie ihre Beziehungen nicht nur objektivieren, sondern diesen Objektivationen - etwa in Gestalt der Wertform der Ware - einen quasi naturhaften Charakter verleihen. Damit erscheinen den Menschen ihre Beziehungen im Sinne eines quasi naturgegebenen Automatismus, den sie nur noch vollziehen können, statt ihn aktiv zu gestalten (vgl. Marx 1890/1977, S. 85ff.).

In einer modernen Terminologie könnte man sagen, dass Marx die kapitalistische Gesellschaft als einen ungeheuren, von Menschen produzierten Softbot begreift, dessen Logik die Beziehungen der Menschen zueinander bestimmt. Die oben beschriebenen Softbots, die Börsenkontrakte eingehen, machen deutlich, was die Verdinglichung der Beziehungen zwischen Käufern und Verkäufern meinen kann. Das Verhältnis dieser Positionen ist derart automatisiert, dass Menschen nicht mehr erforderlich sind, um es zu realisieren. Hier ist es nicht mehr nur so, dass Warenbeziehungen fetischisiert sind, vielmehr funktioniert der Fetisch selbstständig, und es ist fraglich, ob er die Menschen noch braucht. Erste Ansätze dazu kannte Marx bereits in der Entwicklung industrieller Maschinen. Er arbeitet heraus, wie sich die Herrschaft der in der Produktion der Maschine vergegenständlichten Arbeit, d.h. der toten Arbeit, über die lebendige Arbeit durchsetzt. Der Arbeiter wird zum Anhängsel der Maschine (vgl. Marx 1867/1977, Kap. 13).

Der kognitiven Problematisierung der degradierenden Mensch-Maschine-Differenz zufolge gälte es einzusehen, dass Menschen ohnehin wie Maschinen sind. Damit wird insgesamt die Struktur des anthropologischen Quadrats unterminiert - einschließlich der Mensch-Tier-Grenze. Wenn nämlich der Mensch wie eine Maschine zu begreifen ist, müsste dies erst recht für Tiere gelten. Diese Fragerichtung enthält einen performativen Selbstwiderspruch, denn bereits die Feststellung, dass Menschen wie Maschinen sind, enthält eine reflexive Erkenntnis, die gemäß dem Credo ,Menschen sind Maschinen“ eigentlich nicht möglich ist. Dieser Widerspruch wird dadurch aufgelöst, dass in die Gruppe der Menschen eine Zweiteilung eingeführt wird. Danach gibt es einerseits diejenigen Menschen, die zu einer reflexiven Einsicht fähig und folglich keine Maschinen sind. Andererseits gibt es diejenigen Menschen, die wie Maschinen sind und deshalb auch nicht zu einer reflexiven Einsicht in der Lage sind. Bei Letzteren handelt es sich um technisch zu manipulierende Wesen. Der erstgenannten Gruppe, den Reflexionsfähigen, kommt die Aufgabe zu, zum Wohle aller, d.h. auch der maschinell kalkulierbaren Menschen, eine Gesellschaft zu kontrollieren. Die entsprechende Dystopie hat Aldous Huxley (1932) in seinem Werk Schöne neue Welt formuliert. Diese setzt voraus, dass es einige Menschen gibt, die nicht wie Maschinen sind und deshalb eine wissenschaftlich geplante Welt durchsetzen können, in der die maschinenähnlichen Menschen wissenschaftlich durchgeplant und reibungslos funktionieren können. 
Eine Variation dieses Themas findet sich in der neueren Debatte zwischen Neurowissenschaften, Philosophie und Recht (vgl. Krüger 2007; Lampe et al. 2008). Hier geht es um die Frage des freien Willens. Die Neurowissenschaften proklamieren, dass es einen freien Willen nicht geben könne, da der Umweltbezug von menschlichen und tierischen Organismen durch hochkomplexe neuronale Automatismen gesteuert werde (vgl. Roth 2004; Singer 2004). Dies müssten die Philosophie und das Recht einsehen. Es wäre vielleicht sogar unmenschlich, neuronale Automatismen als frei im Sinne des freien Willens anzusehen, denn man würde dadurch menschlichen Organismen etwas zumuten, was diese niemals leisten können (vgl. Pauen 2010, S. 12). Weitere gesellschaftliche Konsequenzen werden in dieser Debatte allerdings nicht gezogen.

Bislang hat - analog zur Mensch-Tier-Unterscheidung - noch jede dramatisierende Infragestellung der Mensch-Maschine-Grenze zu deren Restabilisierung geführt. Aus der Analyseperspektive gesellschaftlicher Grenzregime geht es nicht um die Frage, ob die Infragestellung dieser Grenzen wirklich möglich ist oder nicht. Es geht eher darum, dass eine faktische Infragestellung als ein Indikator für eine grundlegende gesellschaftliche Veränderung zu verstehen wäre.

\subsection{Die Irrealisierung der Grenze zu den Geistwesen}

Die seit der Sattelzeit sich durchsetzende Dramatik der vier anthropologischen Grenzen verdeckt, dass es eine andere Grenze gibt, die in der Moderne weitgehend an Bedeutung verloren hat: die Grenze zwischen Mensch und Geistwesen. Für das vormoderne Europa war dies noch eine praktisch relevante Grenze. Geistwesen wie Teufel oder Dämonen galten als real und diejenigen, die mit ihnen im Bunde standen, wurden als sozial gefährlich angesehen und waren umfangreichen Verfolgungsmaßnahmen ausgesetzt, den Hexenverfolgungen (vgl. Behringer 2001). Im Verlauf des 18. und 19. Jahrhunderts wurde aus der Tatsache, dass es Teufel gibt und Hexen, die mit ihnen im Bunde stehen, ein Aberglaube, der nicht mehr ernst zu nehmen war. Es gibt allerdings auch im Rahmen der modernen Vergesellschaftung Gruppen, die in einem begrenzten Rahmen ein Wirklichkeitsverständnis etablieren, in dem es Hexen, Teufel und Dämonen gibt. Es gibt auch Individuen, in deren Erfahrungswirklichkeit Geister oder Dämonen real vorkommen. Aber es gibt zugleich eine Vielzahl organisatorischer Einrichtungen (Psychiatrie) und psychologisch-psychiatrischer Diskurse, deren Aufgabe darin besteht, ein solches Weltverständnis als nicht allgemein verbindlich zu beschreiben und es damit zu marginalisieren.

Um diese Marginalisierungsprozesse in ihrer Bedeutung $\mathrm{zu}$ erfassen, ist $\mathrm{zu}$ berücksichtigen, dass Menschen im Sinne des anthropologischen Quadrats in normativer Hinsicht eine Sonderstellung haben. Ihnen kommen subjektive Rechte zu. Dies beinhaltet auch das Recht, sich eine eigene Vorstellung von der Welt zu bilden und dieser im Alltag zu folgen. Solange andere Menschen dadurch nicht in Mitleidenschaft gezogen werden, wird menschlichen Individuen in der Moderne ein sehr weitgehendes Recht auf die individuelle Ausgestaltung ihres praktischen Weltverständnisses zugestanden. Menschen können z.B. davon ausgehen, dass sie zur Rettung von Gott und Welt verpflichtet sind und entsprechend handeln. Solange es ihnen zugleich möglich ist, sich an die allgemein verbindlichen Regeln zu halten, stellt dies kein Problem dar (vgl. Lindemann 2018, S. 133f.). 


\section{Modernes Grenzregime und gesellschaftliche Differenzierung}

Die Analyse gesellschaftlicher Grenzregime fördert als Ergebnis zutage, dass auch der natürliche verkörperte Mensch als eine gesellschaftliche Institution zu begreifen ist. Das menschliche Körperindividuum gleich an Freiheit und Würde ist das Resultat einer bemerkenswerten staatlichen Organisationsleistung (mitgliedschaftliche Erfassung der Staatsangehörigen), die ihrerseits an die kirchlichen Organisationsleistungen (Tauf-, Ehe- und Sterbebücher) anschließt und sie weiterentwickelt. Dabei zeigt sich eine interessante historische Pfadabhängigkeit. Der allgemeine Rechtsstatus der geborenen Körperindividuen baut auf dem mit der Taufe etablierten allgemeinen Rechtsstatus der christlichen Seelen auf. Durch die Taufe wurden Neugeborene aus den familiären Zusammenhängen gelöst und der Familie wurde die absolute Macht über die Neugeborenen genommen. Der moderne Staat schließt an diese Organisationsleistung nicht nur an, sondern baut sie mit der Durchsetzung der Beurkundung des Personenstandes (Dokumentation der Geburt, des Ehestandes, des Todes) aus. Der Staat gewährleistet organisatorisch, dass sowohl die Geburt als auch jeder Tod dokumentiert wird. Der Übergang neuer Glieder in die Gesellschaft und aus ihr heraus wird nicht mehr in Form der Taufe, sondern in Form der natürlichen Geburt institutionalisiert, indem er unter die Kontrolle der Medizin gestellt wird (vgl. hierzu insgesamt ebd., Kap. 1).

Genaugenommen findet die Institutionalisierung des menschlichen Körperindividuums im Zusammenspiel zwischen der politisch machtvoll durchgesetzten mitgliedschaftlichen Verfasstheit des Staates, der naturwissenschaftlich orientierten Medizin und der Familie statt. Anders gesagt: Die Familie ermöglicht das Individuum als diesseitigen Körper. Das Leben der Geborenen wird gegen die Familie unmittelbar vom Staat garantiert, der seine Garantenfunktion gegen die Familie vermittels spezialisierter Behörden sowie der Medizin durchsetzt. Letztere bringt ihre eigene Sinnlogik in diese Vermittlung ein, indem sie den Geborenen als biologisch-natürliches Wesen begreift. Damit entsteht eine neue institutionelle Form: das menschliche Körperindividuum mit einem allgemeinen Rechtsstatus, der Freiheit und Würde garantiert. Diese durch den Staat garantierte Institution wird von ihrer Struktur her aber nicht in die Grenzen des Staates eingeschlossen, denn der Staat begrenzt sich im Zugriff auf das menschliche Individuum. Dadurch wird dieses frei, sich in beliebiger Weise zu vergesellschaften. Hier liegt der Zusammenhang zwischen dem modernen gesellschaftlichen Grenzregime und der horizontalen Differenzierung. Menschliche Körperindividuen können sich in beliebiger Weise zu Gruppen zusammenschließen, etwa zu Freundesgruppen, zu Organisationen oder sich zu sozialen Bewegungen zusammenfinden. Sie können unterschiedlich orientierte Handlungszusammenhänge institutionalisieren, wie etwa Wirtschaft oder Wissenschaft. Damit garantiert der Staat ein institutionelles Individuum, welches die Grenzen des Staates in Richtung weltgesellschaftlicher Handlungs- und Kommunikationszusammenhänge überschreiten kann. Der Bürger soll als Mensch weltweit mit allen anderen Menschen kommunizieren, d.h., Handel treiben oder wissenschaftlichen Austausch pflegen können. Deshalb können die Grenzen der Vergesellschaftung nicht mit den Grenzen des Staates identifiziert werden (vgl. ebd., Kap. 2).

Anders gesagt: Die mitgliedschaftliche Verfasstheit von Politik und Recht basiert auf einem nationalstaatlichen Bezug. Im Rahmen des Nationalstaates wird der Bürger zugleich als Mensch verstanden, der sich über den Nationalstaat hinausgehend weltweit vergesellschaften kann. Die weltgesellschaftlich ausgerichtete Sinnorientierung am Menschen übersteigt den Rahmen der mitgliedschaftlich verfassten Handlungsbereiche von Recht und Politik. Indem Politik und Recht eine auf den Menschen hin orientierte Sinngeneralisierung ermöglichen, wird der An- 
schluss an die weltgesellschaftliche Orientierung ermöglicht. Damit wird die Struktur horizontaler Differenzierung ausgebildet. Es entstehen weltgesellschaftlich orientierte Handlungszusammenhänge auf der Grundlage mitgliedschaftlich verfasster Handlungszusammenhänge. Die weltgesellschaftlich orientierten Handlungszusammenhänge können von den mitgliedschaftlich verfassten Zusammenhängen nicht einfach kontrolliert werden. Die Organisationsleistungen von Politik und Recht garantieren die Bedingungen der Weltgesellschaft und die weltgesellschaftlich orientierten Handlungszusammenhänge garantieren den mitgliedschaftlich verfassten diejenigen Orientierungen, die notwendig sind, um die Begrenzungen des Staates zu transzendieren. Ohne den Zwang dazu, die mitgliedschaftliche Verfasstheit zu transzendieren, bestünde die Gefahr, den Menschen total in den Staat zu vereinnahmen. Und umgekehrt würde die weltgesellschaftliche Orientierung ihre Grundlage, den Menschen, zerstören, wenn sie diesen als Weltbürger gegenüber seiner organisatorischen Absicherung im Nationalstaat isolierte (vgl. ebd., Kap. 3).

Im konflikthaften Zusammenspiel zwischen den mitgliedschaftlich verfassten und den weltgesellschaftlich orientierten Handlungszusammenhängen entwickelt sich die moderne Form horizontaler Differenzierung. In diesem Rahmen existieren unterschiedliche Gruppen- und Handlungszusammenhänge nebeneinander. Sie stehen nicht in einem hierarchischen Weisungsverhältnis. Durch Politik und Recht wird zwar ein allgemeiner Rahmen festgelegt, aber was die Fragen des guten moralischen Lebens betrifft, soll auch der Staat den Familien, Freundeskreisen oder Organisationen keine Vorschriften machen dürfen. Es ist erlaubt, sich Ehe- oder Handelspartner außerhalb der nationalstaatlichen Grenzen zu suchen. Wirtschaftsunternehmen sollen ihren Organisationsmitgliedern keine Vorschriften über die Familienbildung machen können und Familien haben keine Weisungsbefugnis gegenüber Freundeskreisen, in denen sich ihre Kinder aufhalten usw.

Der Staat bildet zwar das organisatorische Rückgrat horizontaler Differenzierung, aber da der Handlungsbereich der Politik die Staatsbürger nicht vollständig vereinnahmt, sondern diese als Menschen freisetzt, sich beliebig zu vergesellschaften, bildet sich eine Weltgesellschaft. Deren institutionelle Elemente bilden die menschlichen Körperindividuen gleich an Freiheit und Würde. Besonders das konflikthafte Verhältnis von mitgliedschaftlich verfasster Politik und weltgesellschaftlich orientierter Wirtschaft scheint für die Aufrechterhaltung dieser Differenzierungsform von Bedeutung zu sein. Horizontale Differenzierung beinhaltet ein prekäres Gleichgewicht primär zwischen diesen Handlungs- und Kommunikationszusammenhängen. Das Gleichgewicht bleibt prekär, weil es sowohl auf Seiten der Politik als auch auf Seiten der Wirtschaft die Tendenz gibt, den Menschen vollständig zu vereinnahmen, d.h., den Menschen ausschließlich als ein wirtschaftliches oder politisches Wesen zu bestimmen. Die Grenzen des Sozialen erfahren auf diese Weise eine in sich spannungsvolle Bestimmung. Menschen und von Menschen gebildete Zusammenhänge (etwa Organisationen) sind die staatlich ermöglichten Akteure einer weltweit ausgreifenden Vergesellschaftung.

\section{Forschungsperspektiven}

Die Perspektive gesellschaftlicher Grenzregime eröffnet für die Sozialwissenschaften in mehrfacher Hinsicht Forschungsperspektiven. Zum einen erlaubt es dieses Konzept zu begreifen, dass das menschliche Körperindividuum gleich an Freiheit und Würde eine normativ relevante Institution ist - es ist in keinem Fall als eine natürliche Voraussetzung zu begreifen. Diese 
Einsicht zwingt dazu, die sozialtheoretischen Prämissen der sozialwissenschaftlichen Forschung reflexiv in den Blick zu nehmen. Es erscheint z.B. bis auf Weiteres als unmöglich, handlungstheoretische Konzepte einfach weiter zu verwenden, denn sie setzen das handelnde menschliche Körperindividuum als solches voraus und sind daher untauglich, diesen institutionellen Sachverhalt selbst analytisch in den Blick zu nehmen.

Der Zusammenhang zwischen modernem Grenzregime und horizontaler Differenzierung weist auf den engen Zusammenhang von Nationalstaaten bzw. deren Grenzen und dem moralischen Universalismus des Ethos der Menschenrechte hin (vgl. auch Banse in diesem Band). Hannah Arendt (1949) hatte bereits kurz nach dem Zweiten Weltkrieg auf diesen Zusammenhang aufmerksam gemacht und ihn als praktisches normatives Problem beschrieben. Sie begreift deshalb das Recht gegenüber einem Staat, Rechte geltend machen zu können, als das grundlegende Menschenrecht. Arendt erfasst aber nicht den systematischen Zusammenhang zwischen horizontaler Differenzierung und dem Universalismus der Menschenwürde. Dies wird erst durch die Theorie gesellschaftlicher Grenzregime verständlich. Die kritisch aufgeladene Grenzregimeforschung im Anschluss an Foucault könnte aus diesem Zusammenhang neue Einsichten gewinnen. Der normative Maßstab, dass kein Mensch von einem Leben in Freiheit und Würde auszuschließen sei, spielt einseitig den Universalismus der Menschenwürde gegen die nationalstaatlichen bzw. europäischen Begrenzungspraktiken aus. Damit bleibt unbegriffen, dass zwischen der nationalstaatlichen Grenzziehung und dem moralischen Universalismus der Menschenwürde bzw. der Menschenrechte ein immanenter Zusammenhang besteht. Erst wenn dieser Zusammenhang verstanden wird, kann eine realistische Kritik etwa am „europäischen Grenzregime“ (vgl. Hess et al. 2015) formuliert werden (Lindemann 2018, 385ff.).

Der moralische Universalismus bildet auch den normativen Bezugspunkt für andere Formen der Gesellschaftskritik. Die sozialen Grenzen, die etwa Arbeiter, Frauen oder Schwule, Lesben sowie andere Minderheitengruppen von einer allgemeinen gesellschaftlichen Teilhabe ausgeschlossen haben, wurden im Namen des moralischen Universalismus der Menschenrechte von sozialen Bewegungen kritisiert und mehr oder weniger erfolgreich verändert. Die Arbeiterbewegung des 19. und 20. Jahrhunderts kämpfte dafür, dass Arbeiter als Menschen nicht vollständig von der Wirtschaft vereinnahmt werden können. Die Frauenbewegung stritt dafür, dass die Existenz von Frauen nicht auf die Familie beschränkt werden dürfe. Stattdessen sollten sie als Menschen das Recht haben, sich in einer individuell bestimmten Weise zu vergesellschaften. Die Kämpfe der Emanzipationsbewegungen zeigen, dass das gesellschaftliche Grenzregime der europäisch-nordamerikanischen Moderne einen moralischen Überschuss aufweist, der eine allgemeine Kritik an gesellschaftlichen Grenzziehungen ermöglicht (vgl. Bruns in diesem Band). Hieraus ergibt sich eine neue gesellschaftstheoretisch orientierte Perspektive auf soziale Bewegungen (vgl. Lindemann 2018, Kap. 3). Soziale Bewegungen werden durch das moderne Grenzregime ermöglicht und diese Bewegungen erzeugen bzw. stabilisieren die Bedingungen horizontaler Differenzierung, indem sie dafür eintreten, dass die menschlichen Körperindividuen nicht einseitig durch die Logik einzelner Gruppen- oder Handlungszusammenhänge bestimmt werden.

Weiterhin ergeben sich in der Perspektive gesellschaftlicher Grenzregime neue Möglichkeiten des Kulturvergleichs, indem untersucht wird, welche Arten von Akteuren in welcher Weise ein normativer Status zukommt. Die Besonderheiten des christlichen europäisch-nordamerikanischen Wegs in die horizontale Differenzierung, d.h. in die Moderne, lassen sich in einer grenzregimetheoretischen Perspektive besser erfassen. Das christliche Abendland war bereits 
über Jahrhunderte durch eine große Organisationsleistung geprägt, für die es in anderen Glaubensrichtungen kein Äquivalent gibt. Weder der Islam noch der Buddhismus haben eine der verpflichtenden Kindstaufe vergleichbare Institution entwickelt. Dies erlaubt einen neuen Blick auf die unterschiedlichen Wege in die Moderne und führt auf die Frage, ob es technologische Hochentwicklung unabhängig von horizontaler Differenzierung und der damit einhergehenden Orientierung am Ethos der Menschenrechte geben kann.

Es ist wahrscheinlich nicht auszuschließen, dass es im Rahmen der gegenwärtigen Vergesellschaftung miteinander konkurrierende Grenzregime gibt. Dies führt u.a. zu neuen religionssoziologischen Fragen: Wie koordinieren etwa streng gläubige Christen oder Muslime ihre religiös eingehegten Grenzregime, in deren Rahmen es Engel oder Geister geben mag, mit dem modernen Grenzregime? Im Rahmen einer solchen Perspektive würden die existenziellen Konflikte, in die Gläubige in modernen Gesellschaften gezwungen werden, deutlicher zutage treten.

\section{Weiterführende Literatur}

Evans, E. P. (1906): The Criminal Prosecution and Capital Punishment of Animals. London/Boston: Faber and Faber.

Luckmann, Thomas (1970): On the Boundaries of the Social World. In: Natanson, Maurice (Hrsg.): Phenomenology and Social Reality. Essays in Memory of Alfred Schutz. The Hague: Nijhoff, S. 73-100.

Lüdtke, Nico (2015): Das Soziale ohne Grenzen? Eine historisch-wissenssoziologische Analyse zu den Grenzen der Sozialwelt in der Frühen Neuzeit. Weilerswist: Velbrück Wissenschaft.

Lindemann, Gesa (2014): Weltzugänge. Die mehrdimensionale Ordnung des Sozialen. Weilerswist: Velbrück Wissenschaft.

Lindemann, Gesa (2018): Strukturnotwendige Kritik. Theorie der modernen Gesellschaft, Bd. 1. Weilerswist: Velbrück Wissenschaft.

\section{Literaturverzeichnis}

Arendt, Hannah (1949): Es gibt nur ein einziges Menschenrecht. In: Die Wandlung 4, S. 754-770.

Beck, Susanne (2016): The Problem of ascribing legal responsibility in the case of robotics. AI \& Society 31, H. 4, S. 473-481.

Behringer, Wolfgang (2001): Heinrich Kramers „Hexenhammer“: Text und Kontext. In: Schmauder, Andreas (Hrsg.): Frühe Hexenverfolgung in Ravensburg und am Bodensee. Konstanz: UVK, S. 83-124.

Berger, Peter Ludwig/Luckmann, Thomas (1966/1980): Die gesellschaftliche Konstruktion der Wirklichkeit. Eine Theorie der Wissenssoziologie. Frankfurt/M.: Fischer.

Bergmann, Anna (2005): Der entseelte Patient. Die moderne Medizin und der Tod. Berlin: Aufbau.

Büsing, Sarah Marie (2021): Die Erdeutung von Wille und Wohl bei medizinisch-ethischen Behandlungsentscheidungen in der Frühgeborenenintensivmedizin in Deutschland und der Schweiz. Dissertation an der Carl von Ossietzky Universität Oldenburg.

Cavalieri, Paola/Singer, Peter (Hrsg.) (1993/1996): Menschenrechte für die großen Menschenaffen. München: Goldmann.

Darwin, Charles (1871): The descent of man and selection in relation to sex, 2 Volumes. London: Murray.

Descola, Philippe (2005/2011): Jenseits von Natur und Kultur. Frankfurt/M.: Suhrkamp.

Duden, Barbara/Schlumbohm, Jürgen/Veit, Patrice (Hrsg.) (2002): Geschichte des Ungeborenen. Zur Erfahrungs- und Wissenschaftsgeschichte der Schwangerschaft. Göttingen: Vandenhoeck \& Ruprecht.

Durkheim, Émile (1950/1999): Physik der Sitten und des Rechts, Frankfurt/M.: Suhrkamp

Foucault, Michel (1963/1988): Die Geburt der Klinik. Eine Archäologie des ärztlichen Blicks. Frankfurt/M.: Fischer.

Foucault, Michel (1975/1979): Überwachen und Strafen. Die Geburt des Gefängnisses. Frankfurt/M: Suhrkamp.

Fuchs, Richard (1996): Tod bei Bedarf. Das Mordsgeschäft mit der Organtransplantation. Frankfurt/M./ Berlin: Ullstein.

Graumann, Sigrid (2012): Die Geburt als Grenze zur Konstitution sozialer Personen: Ein soziologischtheoretischer Beitrag zur bioethischen Diskussion über Spätabbrüche und die Behandlung von Frühchen. In: Joerden, Jan/Hilgendorf, Eric/Petrillo, Natalia/Thiele, Felix (Hrsg.): Menschenwürde in der Medizin: Quo vadis? Baden Baden: Nomos, S. 13-32. 
Hess, Sabine/Kasparek, Bernd/Schwertl, Magdalena/Sontowski, Simon (2015): Europäisches Grenzregime. Einleitung zur ersten Ausgabe. In: movements. Journal for Critical Migration and Border Regime Studies 1, H. 1, online: http://movements-journal.org/issues/01.grenzregime/02.einleitung.html, 18.05.2019.

Huxley, Aldous (1932): Brave new world. Ditzingen: Reclam.

Kelsen, Hans (1946/1982): Vergeltung und Kausalität. Wien/Köln/Graz: Böhlau.

Kessel, Martina (2001): Die Angst vor dem Scheintod im 18. Jahrhundert. Körper und Seele zwischen Religion, Magie und Wissenschaft. In: Schlich, Thomas/Wiesemann, Claudia (Hrsg.): Hirntod. Zur Kulturgeschichte der Todesfeststellung. Frankfurt/M.: Suhrkamp, S. 133-166.

Klages, Ludwig (1913/2006): Mensch und Erde. Bonn: Bouvier.

Klinkhammer, Gisela (1997): Entschieden gegen eugenische Tendenzen. Kirchenwort zur pränatalen Diagnostik. Deutsches Ärzteblatt 94, H. 22, S. 1485.

Krüger, Hans Peter (Hrsg.) (2007): Deutsche Zeitschrift für Philosophie, Sonderband 15: Hirn als Subjekt. Philosophische Grenzfragen der Neurobiologie. Berlin: Akademie.

Lampe, Ernst-Joachim/Pauen, Michael/Roth, Gerhard (Hrsg.) (2008): Willensfreiheit und rechtliche Ordnung. Frankfurt/M.: Suhrkamp.

Lindemann, Gesa (1993/2011): Das paradoxe Geschlecht. Transsexualität im Spannungsfeld von Körper, Leib und Gefühl. Wiesbaden.

Lindemann, Gesa (2003): Beunruhigende Sicherheiten. Zur Genese des Hirntodkonzepts. Konstanz: Universitätsverlag.

Lindemann, Gesa (2018): Strukturnotwendige Kritik. Theorie der modernen Gesellschaft, Bd. 1. Weilerswist: Velbrück Wissenschaft.

Lindemann, Gesa (2020): Die Ordnung der Berührung. Staat, Gewalt und Kritik in Zeiten der Coronakrise. Weilerswist: Velbrück Wissenschaft.

Luckmann, Thomas (1970): On the Boundaries of the Social World. In: Natanson, Maurice (Hrsg.): Phenomenology and Social Reality. Essays in Memory of Alfred Schutz. The Hague: Nijhoff, S. 73-100.

Lüdtke, Nico (2015): Das Soziale ohne Grenzen? Eine historisch-wissenssoziologische Analyse zu den Grenzen der Sozialwelt in der Frühen Neuzeit. Weilerswist: Velbrück Wissenschaft.

Luhmann, Niklas (1965/1999): Grundrechte als Institution. Berlin: Duncker und Humblot.

Luhmann, Niklas (1971/2005): Die Weltgesellschaft. In: Ders.: Soziologische Aufklärung 2. Aufsätze zur Theorie der Gesellschaft. Wiesbaden: VS, S. 63-88.

Luhmann, Niklas (1972): Rechtssoziologie, 2. Bde., Reinbek bei Hamburg: Rowohlt.

Luhmann, Niklas (1997): Die Gesellschaft der Gesellschaft, 2 Bde. Frankfurt/M.: Suhrkamp.

Marx, Karl (1867/1977): Das Kapital, Bd. 1. Berlin: Dietz.

Matsuzaki, Hironori/Lindemann, Gesa (2016): The autonomy-safety-paradox of service robotics in Europe and Japan - a comparative analysis. In: AI \& Society 31, H. 4, S. 501-517.

Matsuzaki, Hironori/Straub, Ilona/Lindemann, Gesa (2016): Going beyond the laboratory. Social robotics in everyday-life. In: AI \& Society 31, H. 4, S. 441-444.

Matthias, Andreas (2004): The responsibility gap: Ascribing responsibility for the actions of learning automata. In: Ethics and Information Technology 6, H. 3, S. 175-183.

Meyer, Christoph H.F. (2013): Taufe und Person im ersten Jahrtausend Beobachtungen zu den christlichen Wurzeln einer Grundkategorie europäischen Rechtsdenkens. In: Rechtsgeschichte 21, S. 89-117.

Neumann, Almut (2007): Teufelsbund und Teufelspakt (Mittelalter). In: Gersmann, Gudrun/Moeller, Katrin/Schmidt, Jürgen-Michael (Hrsg.): Lexikon zur Geschichte der Hexenverfolgung. http://www.historic um.net/no_cache/persistent/artikel/5527/, 07.12.2007.

Parsons, Talcott (1971/1985): Das System moderner Gesellschaften. Weinheim, München: Juventa.

Pauen, Michael (2010): Einleitung: Teil I: Philosophische und psychologische Beiträge. In: Lampe, ErnstJoachim/Pauen, Michael/Roth, Gerhard (Hrsg.): Willensfreiheit und rechtliche Ordnung. Frankfurt/M.: Suhrkamp, S. 9-16.

Pernick, Martin S. (1988): Back from the Grave: Recurring Controversies over Defining and Diagnosing Death in History. In: Zaner, Richard M. (Hrsg.): Death: Beyond Whole-brain Criteria. Dordrecht, Boston, London: Kluwer, S. 17-74.

Roth, Gerhard (2004): Worüber dürfen Hirnforscher reden - und in welcher Weise. Deutsche Zeitschrift für Philosophie 52, H. 2, S. 221-234.

Rothhaar, Markus/Hähnel, Martin/Kipke, Roland (Hrsg.) (2017): Der manipulierbare Embryo. Paderborn: mentis.

Rüve, Gerlind (2008): Scheintod. Zur kulturellen Bedeutung der Schwelle zwischen Leben und Tod um 1800. Bielefeld: transcript.

Said, Edward (1978): Orientalism. New York: Pantheon Books. 
Schaeffer, Katja (2021): Behandlungsentscheidungen bei Frühgeborenen - eine theoriegeleitete, ethnographische Untersuchung. Dissertation an der Carl von Ossietzky Universität Oldenburg.

Singer, Wolf (2004): Selbsterfahrung und neurobiologische Fremdbeschreibung. Zwei konfliktträchtige Erkenntnisquellen. Deutsche Zeitschrift für Philosophie 52, H. 2, S. 235-255. 